\title{
Tuning of the lateral specific force gain based on human motion perception in the Desdemona simulator.
}

\author{
Bruno Jorge Correia Grácio*, M. M. (René) van Paassen ${ }^{\dagger}$ and Max Mulder ${ }^{\ddagger}$ \\ Delft University of Technology, Delft, The Netherlands \\ Mark Wentink ${ }^{\S}$ \\ TNO Defense, Security and Safety, Soesterberg, The Netherlands
}

\begin{abstract}
Generally, motion simulators present motion and visual cues different from each other due to the physical limitations of the motion platform. Nonetheless, high fidelity motion platforms are capable of simulating some maneuvers one-to-one, i.e., motion cues equal to visual cues. However, one-to-one simulation is normally not preferred by subjects and the simulator motion is reported as too strong. In this study we investigated whether this overestimation depends on the frequency and amplitude of inertial motion. The stimuli in this study consisted of translations in the lateral direction. The Desdemona research simulator was used to generate the motion profiles. Six sinusoidal profiles with different combinations of amplitude and frequency were used as reference stimuli. For every experimental condition, the visual and inertial information had equal frequency but different amplitude. Subjects had to change the inertial motion amplitude until they obtained the best relation between the two sources of motion information. Our results showed that stimuli with high amplitude were associated with smaller motion gains than stimuli with lower amplitude. The same occurred for stimuli with higher frequency when compared to stimuli with lower frequency. The findings in this study suggest that a dynamic scaling algorithm for inertial motion could improve the perceived realism of motion simulation.
\end{abstract}

\section{Introduction}

In motion simulators subjects can be presented with sensory cues that mimic the sensory stimulation that is experienced in a real vehicle. Part of this sensory information is provided by inertial cues generated by a motion platform. However, most vehicle motions cannot be generated in the simulator because of its physical limits. Therefore, algorithms are needed to transform vehicle motion into simulator motion. Such algorithms are usually referred to as Motion Cueing Algorithms (MCA). New high fidelity motion platforms make it possible to simulate certain maneuvers one-to-one, i.e. the simulator motion is equal to the vehicle motion. Examples of these large amplitude platforms are the Desdemona Research simulator at TNO Human Factors in the Netherlands and the KUKA simulator at Max Planck Institute in Germany. However, several studies ${ }^{1-4}$ showed that subjects perceive one-to-one motion in a simulator differently than in real life. Subjects in these studies reported motion to be too strong. Feenstra et al. ${ }^{1}$ and Pretto et al. ${ }^{2}$ conducted a car driving study where subjective measurements showed that simulator motion needed to be scaled down in order to subjectively match "what was seen with what was felt". In these studies a preferred scaling factor of the motion between 0.5 and 0.7 was reported. These values were obtained from paired comparisons between motion filters with different characteristics. Groen et al. ${ }^{3}$ showed that a simulated take-off maneuver was experienced as more realistic when translational accelerations were scaled

*Ph.D. student, Faculty of Aerospace Engineering, Control and Simulation Division, B.J.CorreiaGracio@tudelft.nl, P.O. Box 5058, 2600 GB Delft, The Netherlands.

${ }^{\dagger}$ Associate Professor, Faculty of Aerospace Engineering, Control and Simulation Division, M.M.vanPaassen@tudelft.nl, P.O. Box 5058, 2600 GB Delft, The Netherlands, AIAA Member

¥Professor, Faculty of Aerospace Engineering, Control and Simulation Division, M.Mulder@tudelft.nl, P.O. Box 5058, 2600 GB Delft, The Netherlands, AIAA Member

$\S$ Researcher, Perception and Simulation, mark.wentink@tno.nl, P.O. Box 23, 3769 ZG Soesterberg, The Netherlands 
down. Again a preferred motion gain of 0.7 was found. In another study by Groen et al., ${ }^{4}$ it was shown that for a correct percept of a decrab maneuver in a simulator, the simulated sway motion had to be smaller than the actual aircraft motion. These studies directly compared the motion cues in the simulator with the motion cues of the real vehicle. Therefore, errors in the vehicle model used to generate the correct aircraft motion could influence the percept of the motion cues in the simulator. Another factor that can influence the subjects' motion judgment is the fact that the vehicle model handles differently than the vehicle subjects are used to in the real world. In the studies mentioned above, the authors did not have the objective to find a relation of the motion gain with the amplitude and frequency of the vehicle motion signal. The goal of the present study is to investigate whether the motion gain used in MCA for linear accelerations depends on the frequency and amplitude of the input motion signal.

The Desdemona research simulator was used as a platform to study which motion gain would give the best match between visual and inertial cues. Subjects were presented with lateral translational movements, where they had to match the inertial information with the visual information. The use of lateral cues allows for the comparison of the obtained scaling values with previous experiments conducted in Desdemona ${ }^{1,5,6}{ }^{\text {where }}$ similar motion profiles were used. The visual information was displayed via the simulator's projectors while the inertial information was generated using the motion platform. Both signals were sinusoids with matching phase and frequency, but different amplitudes. We used six different visual profiles, each with a different combination of frequency and amplitude. The amplitude/frequency values were a compromise between the simulator limitations and human motion perception ranges that are of interest for vehicle simulation. In order to decrease the experiment duration, we developed an online tuning method where subjects could change the inertial motion amplitude in-the-loop. Classically, tuning of MCAs is conducted with an experienced subject that verbally indicates to the MCA designer how the motion is being perceived, while the designer tries to achieve the experienced subject requirements by changing values in the MCA. With our method we give full control of the tuning to the subject inside the cabin by means of a joystick. A joystick's deflection would change the inertial amplitude while the subject is experiencing it, making it easier to decide whether the experienced motion is too strong or too weak. The controlled signal was the acceleration of the motion platform since it is the signal sensed by the vestibular system. ${ }^{7}$

The following sections describe in more detail the experimental protocol and present the experimental results. At the end, we discuss the results and present the conclusions of this study.

\section{Method}

The experimental goal was to study the relation between the inertial and visual motion amplitude that creates a realistic percept in the Desdemona simulator. The dependent variable of this study was the motion gain, which is defined by the ratio between the inertial and visual amplitude. The visual signals were designed with different frequencies and amplitudes to determine whether there was an effect of these variables on the motion gain. Therefore, the independent variables for this study were the visual amplitude, the visual frequency and the initial motion gain, which could be higher or lower than one.

\section{II.A. Apparatus}

We used the Desdemona research simulator (Figure 1) located at the TNO institute in Soesterberg, the Netherlands. The simulator features a centrifuge based design with six degrees-of-freedom (DoF). More details regarding the motion platform can be found in. ${ }^{8}$ For this study, we only used the 8-meter horizontal track of the simulator to generate lateral motion cues. The lateral motion stimuli were sinusoidal acceleration profiles.

The simulator cabin contains a generic F-16 cockpit with realistic throttle, side-stick and rudder pedals. For this study only the side-stick was used. Three beamers projecting on a three part flat screen generate the Out-The-Window (OTW) visual. The Field-of-View (FOV) was 120 degrees horizontal and 32 degrees vertical. The participants were seated approximately at 1.5 meters from the simulator screens. The OTW view showed the aircraft-parking zone of the Innsbruck airport in Austria. A yellow car was parked in front of the terminal as shown in Figure 2. A realistic visual scene was chosen because it resembles the type of scenes normally displayed in vehicle simulation. 


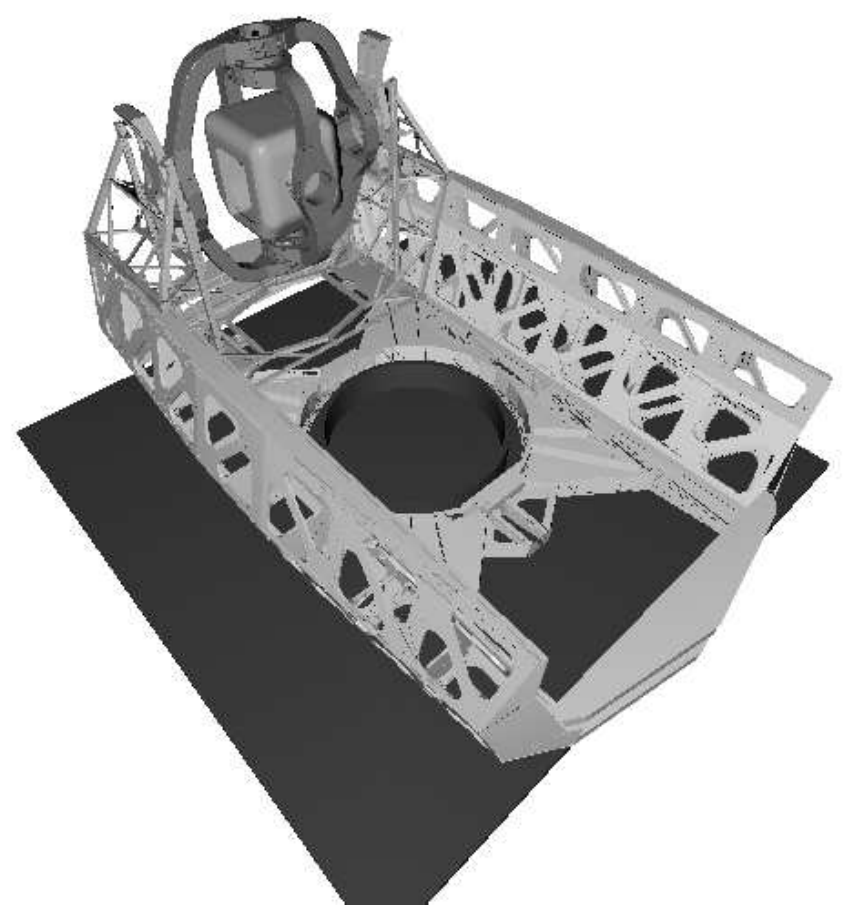

Figure 1. Schematic of the Desdemona research simulator developed by AMST Systemtechnik (Austria) and TNO Human Factors (Netherlands).

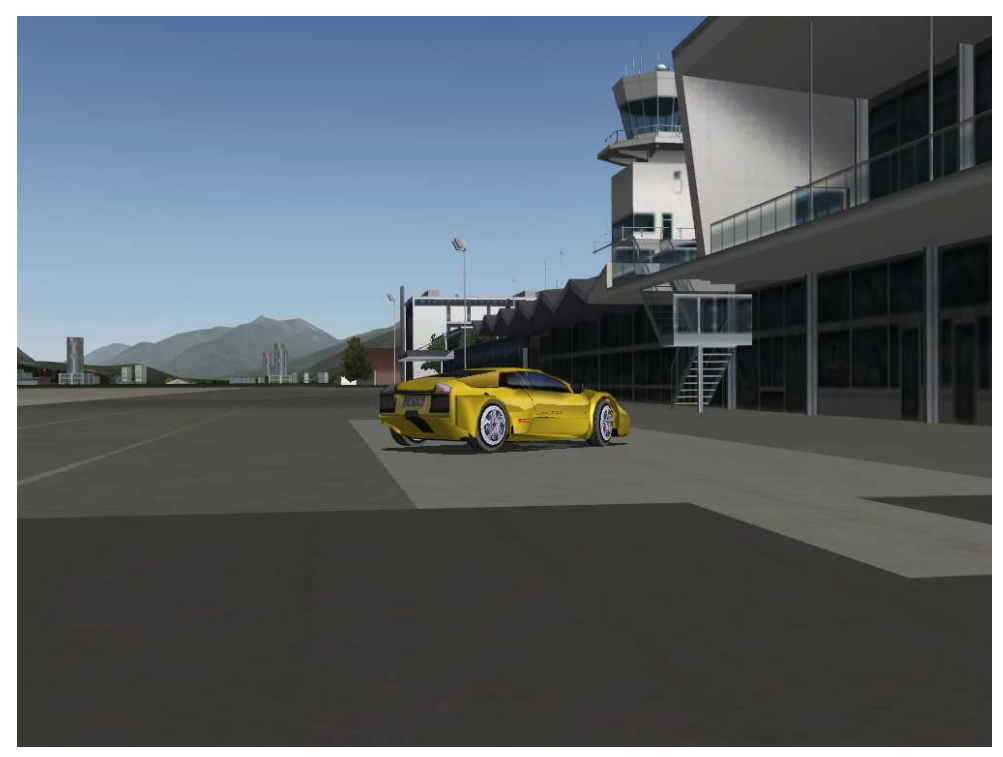

Figure 2. Visual scene showing part of the Innsbruck airport in Austria.

\section{II.B. Subjects}

Twelve subjects, five males and seven females, participated in this experiment. Their average age was 28 years with a standard deviation of 11 years. All subjects were TNO employees. An informed consent was signed before participation. Subjects sat in the chair and wore a headset that outputted white noise to mask actuator sound. This headset was also used for communication between the participant and the experimenter. Participants had to adjust the motion amplitude online using the side-stick inside the cabin until the best match between "what they were feeling and what they were seeing" was achieved. When finished, they communicated to the experimenter how confident they were that the motion gain they chose 
was the best match they could achieve with the visual being displayed.

\section{II.C. Experimental Design}

The experiment had a repeated measures design. The amplitude of the inertial acceleration signal was measured for six different visual signals. The visual signals were combinations of sinusoids with amplitudes of 1 and $2.5 \mathrm{~m} / \mathrm{s}^{2}$ and frequencies of $0.2,0.4$ and $0.8 \mathrm{~Hz}$. The frequency values were chosen near the zone where there is an increase of sensitivity of the otolith model described by Hosman. ${ }^{9}$ Each visual signal was measured two times, one for initial inertial amplitudes higher than the visual amplitude and other for initial inertial amplitudes lower than the visual amplitude. This was done to test whether a different initial condition would produce different motion gains for the same visual signal. This led to initial motion gains of 1.4 and 0.6 that were obtained based in the motion platform limitations. The frequency of the inertial signal was always equal to the one of the visual signal. The independent variables combinations make a total of twelve different experimental conditions. Each experimental condition was repeated three times, meaning that subjects performed 36 simulator runs. A Latin squares design was used to randomize the experimental conditions for each repetition. This means that for each repetition, all twelve experimental conditions were performed in a different order for all twelve subjects. With this we expect to reduce any order effect that may affect the motion gain.

\section{II.D. Procedure}

Subjects had to adjust the motion gain of the simulator until the best match between the visual and inertial cues was obtained. After being seated in the simulator, practice conditions were run to ensure that the subjects understood the task. Each experimental run started by pressing the trigger-button of the side-stick. Then, the visual moved with the same frequency of the motion platform but with different amplitude. The initial motion gain depended of the experimental condition. The visual amplitude remained constant during the experimental run but the inertial amplitude could be changed. The side-stick was used to actively change the inertial amplitude. A deflection to the left would decrease the inertial amplitude while a right-deflection would increase it. Subjects were instructed to adjust the inertial amplitude to the visual amplitude until the cues matched optimally. Subjects were encouraged to try out different inertial values before settling for one. When satisfied with their adjustment, the trigger-button was pressed to stop the experimental run. Subjects were then asked to rate how confident they were that the obtained inertial amplitude was optimal. A value ranging from one to ten was assigned, where one is not confident and ten is highly confident. Each participant performed the 36 experimental runs such that the experimental conditions order was not repeated between subjects.

\section{II.E. Data analysis}

We defined the motion gain as the ratio between the motion and visual acceleration signal. A gain of one represents the real life situation where the visual cues are equal to the inertial cues. A motion gain higher than one shows that subjects wanted inertial cues stronger than the visual cues. A gain lower than one shows a preference for inertial cues weaker than the visual cues.

To analyze the data, we calculated for every subject the mean gain of every experimental condition. After, we used these individual means to calculate the total mean for every experimental condition. A repeated measures ANOVA was used to determine if the mean motion gains obtained for every experimental condition were statistically different from each other. The statistical analysis were performed using PASWS 18.0.

\section{Results}

\section{III.A. Motion gain}

Figure 3 shows the mean motion gain values for all experimental conditions. The results were pooled for the three repetitions since no significant differences were found between them. The results from the statistical analysis are shown in Table 1. The mean motion gain was higher when the experimental trial started with a motion gain higher than one. A repeated measures ANOVA showed that this difference between initial 
conditions was significant. The added mean motion gain for the initial condition dependent variable were respectively 0.89 and 0.65 for the initial motion gain higher than one and initial motion gain lower than one.

The motion gain was also dependent on the visual amplitude. A repeated measures ANOVA showed that the condition with lower visual amplitude had significantly higher motion gains than the condition with higher visual amplitude. The added means showed a motion gain of 0.90 and 0.65 respectively for the 1.0 $\mathrm{m} / \mathrm{s}^{2}$ and $2.5 \mathrm{~m} / \mathrm{s}^{2}$ visual amplitudes.

The statistical tests showed a significant main effect of the frequency in the motion gain. The pooled mean motion gains were $0.90,0.81$ and 0.61 respectively for the $0.2,0.4$ and $0.8 \mathrm{~Hz}$ experimental conditions. A post hoc test using a Bonferroni correction showed that the mean gains for the $0.8 \mathrm{~Hz}$ experimental conditions were significantly higher than the $0.2 \mathrm{~Hz}(\mathrm{p}=0.011)$ and the $0.4 \mathrm{~Hz}(\mathrm{p}=0.008)$ experimental conditions.

A significant interaction between stimulus amplitude and initial condition on the motion gain was observed. Figure 3 shows that the effect of the initial condition on the motion gain was higher for the low amplitude signals than for the high amplitude signals. For the low amplitudes, we obtained a motion gain with a grand mean of 1.1 and 0.74 respectively for an initial condition higher and lower than one. For the high amplitudes, the total means were 0.73 for an initial condition higher than one and 0.57 for an initial condition lower than one.

A significant interaction was also found between the initial conditions and the frequency content of the signal. From Figure 3, one can observe for the conditions starting with motion gain higher than one, a greater decrease of the obtained mean motion gain from the lower to the higher frequencies than for the conditions starting with a motion gain lower than one. This is especially visible for the conditions with a frequency of $0.2 \mathrm{~Hz}$ when compared with the conditions with a frequency of $0.8 \mathrm{~Hz}$. For the conditions starting with a motion gain higher than one, the mean values were 1.06, 0.94 and 0.68 respectively for the $0.2,0.4$ and 0.8 $\mathrm{Hz}$ conditions. For the conditions with an initial motion gain lower than one, the mean values were 0.74 , 0.69 and 0.53 respectively for the $0.2,0.4$ and $0.8 \mathrm{~Hz}$ conditions.

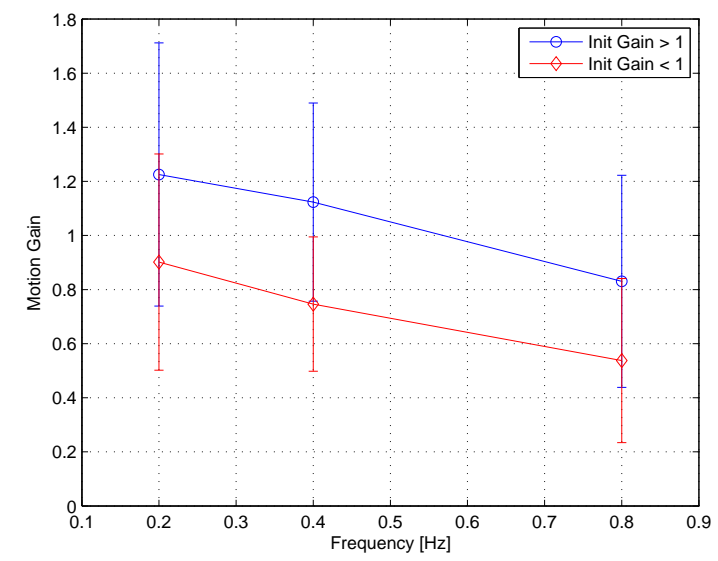

(a) Visual Amplitude $=1 \mathrm{~m} / \mathrm{s}^{2}$

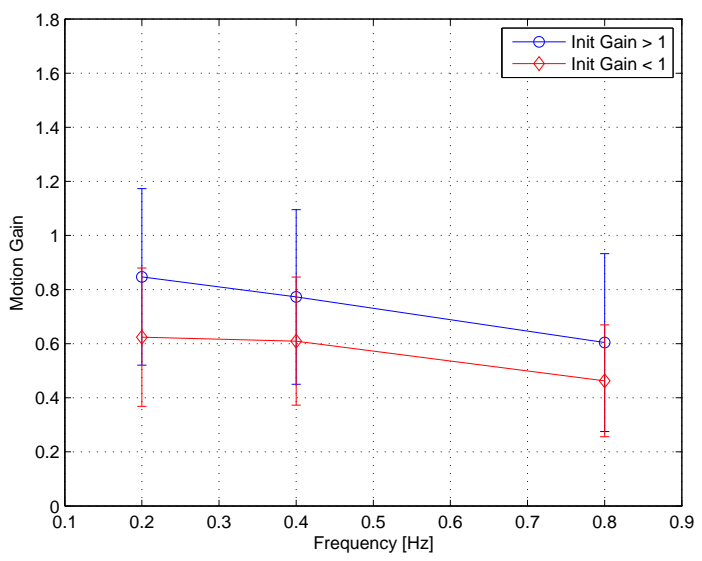

(b) Visual Amplitude $=2.5 \mathrm{~m} / \mathrm{s}^{2}$

Figure 3. Mean motion gain for the $1 \mathrm{~m} / \mathrm{s}^{2}$ amplitude visual signal (left) and $2.5 \mathrm{~m} / \mathrm{s}^{2}$ amplitude visual signal (right). The vertical bars represent the $95 \%$ confidence intervals.

Table 1. Repeated measures ANOVA results for the mean phase difference $(* *=p<0.01 ; *=0.01 \leq p<0.05)$.

\begin{tabular}{ccccc}
\hline \hline Independent Variables & Correction & F-ratio & $p$ & sig. \\
\hline Amplitude & - & $\mathrm{F}(1,11)=19.61$ & 0.001 & $* *$ \\
Initial Condition & - & $\mathrm{F}(1,11)=23.33$ & 0.001 & $* *$ \\
Frequency & Greenhouse-Geisser & $\mathrm{F}(1.20,13.16)=12.66$ & 0.002 & $* *$ \\
Amplitude $*$ Initial Condition & - & $\mathrm{F}(1,11)=15.48$ & 0.002 & $* *$ \\
Initial Condition * Frequency & - & $\mathrm{F}(2,22)=4.85$ & 0.018 & $*$ \\
\hline \hline
\end{tabular}




\section{III.B. Confidence values}

Figure 4 shows the mean confidence values obtained after each experimental condition. The results show that subjects were quite confident in their reports. Nevertheless, a small decay in the confidence values for the highest frequency conditions was noticeable. A repeated measures ANOVA was used as an indication of the statistical significance of this drop in the subjects' confidence. We found a significant main effect of the frequency on the confidence levels, $\mathrm{F}(2,22)=13.40, \mathrm{p}=0.000$. The post hoc tests revealed that the 0.8 $\mathrm{Hz}$ conditions had a mean confidence level significantly lower than the $0.2 \mathrm{~Hz}(\mathrm{p}=0.004)$ and $0.4 \mathrm{~Hz}(\mathrm{p}=$ $0.004)$ conditions. The pooled mean confidence values were $7.6,7.4$ and 6.7 respectively for the $0.2,0.4$ and $0.8 \mathrm{~Hz}$ conditions.

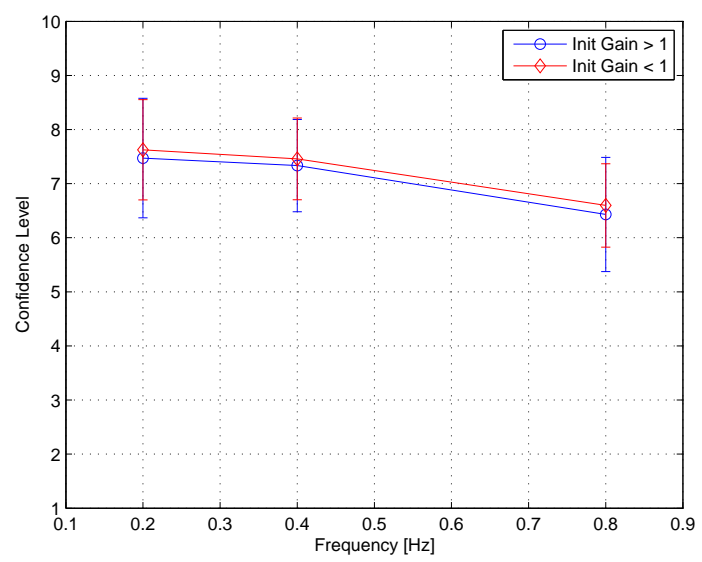

(a) Visual Amplitude $=1 \mathrm{~m} / \mathrm{s}^{2}$

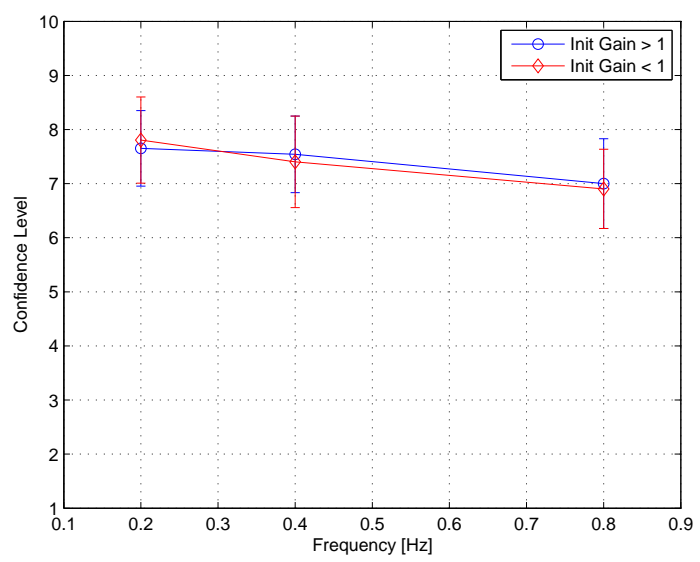

(b) Visual Amplitude $=2.5 \mathrm{~m} / \mathrm{s}^{2}$

Figure 4. Mean confidence levels for the $1 \mathrm{~m} / \mathrm{s}^{2}$ amplitude visual signal (left) and $2.5 \mathrm{~m} / \mathrm{s}^{2}$ amplitude visual signal (right). The vertical bars represent the $95 \%$ confidence intervals.

\section{Discussion}

\section{IV.A. Motion gain dependence on stimulus amplitude}

The results showed that with an increase of the stimulus amplitude, the subjective motion gain decreased. We do not know how the motion gain varies as a function of the stimulus amplitude, it can be a linear/nonlinear dependence; it can saturate for stimuli with higher amplitudes. However, we can state that these results are interesting for the development of MCAs. From a MCA design point of view, it is beneficial that for higher amplitudes subjects prefer a motion gain lower than one. This means that smaller simulator excursions could be used for stimuli with higher amplitude without loss of perceived realism to the subject. Based on these results, a scaling algorithm can be designed where the used motion gain decreases with the increase of the reference motion stimuli. This reduction of the necessary simulator motion envelope is valuable for generating motion cues that could not be produced before due to the use of a constant scaling algorithm. To the authors' knowledge, there are no MCAs that dynamically scale the motion information based in the amplitude of the reference motion stimuli.

The mean motion gain values obtained are close to the values obtained in literature ${ }^{2-4}$ and on previous Desdemona studies. ${ }^{1,5,6}$ Groen et al. ${ }^{3}$ when simulating a take off run found a preferred motion gain of 0.2 for the surge motion of the simulator. The amplitude of the reference signal was $3.5 \mathrm{~m} / \mathrm{s}^{2}$, which is higher than what was used in this experiment. The low value found for their surge motion filter may be explained by the present observation that the higher the amplitude of the motion signals, the lower the motion gain preferred by subjects. Feenstra et al. ${ }^{1}$ found that subjects preferred a motion gain of 0.7 when driving trough a slalom course that delivered a theoretical lateral specific force of approximately $1.2 \mathrm{~m} / \mathrm{s}^{2}$. Their results are also within the range of our findings. 


\section{IV.B. Motion gain dependence on stimulus frequency}

The subjective motion gain was affected by the frequency of the motion signal. Signals with higher frequency tended to have a motion gain smaller than signals with lower frequencies. For a sinusoidal motion profile, an increase in frequency also means an increase in the signal jerk. This means that for profiles with the same acceleration amplitude but different frequencies, the jerk will be higher for the stimulus with the highest frequency. The decrease of the motion gain with the frequency indicates that humans are sensitive to high jerk, rating then motion as too strong. ${ }^{10}$ Human sensitivity to jerk was already discussed to be an important motion perception issue in other studies. ${ }^{10-12}$ Grant et al. ${ }^{10}$ showed that the concept of motion strength depended not only on the acceleration value of the motion signal but also on the jerk value. Therefore, it is incorrect not to consider the jerk sensitivity when modeling the linear motion perception system. Some authors model the otolith as a unit gain block where the input is specific force. This modeling does not take into account a jerk sensitivity. On the other hand, Hosman ${ }^{9}$ included high frequency sensitivity in the otolith model. However, it is yet not clear whether the jerk sensitivity comes from the otolith, from the somatosensory system or both.

Just as a qualitatively measurement, we decided to plot the obtained motion gains against the transfer function of the otolith proposed by Hosman. ${ }^{9}$ Eq. 1 shows the otolith transfer function, where $\tau_{L}$ is the lead-time constant, $\tau_{1}$ is the main time constant and $\tau_{2}$ the secondary time constant. Wentink et al. ${ }^{13}$ set these time constants in their motion perception toolbox as $0.3,0.12$ and 0 respectively for $\tau_{L}, \tau_{1}$ and $\tau_{2}$.

$$
H_{O T O}(s)=\frac{\left(1+\tau_{L} s\right)}{\left(1+\tau_{1} s\right)\left(1+\tau_{2} s\right)}
$$

The motion gain values were inverted to be compatible with the information given by the transfer function, i.e. if the motion gain is 0.8 , the sensitivity value would be 1.25 . This means that subjects decreased the motion gain when they felt that the perceived motion was too strong when compared with the visual information being displayed. Figure 5 shows the mean sensitivity plotted against the otolith model. We noticed that for both signal amplitudes, the obtained subjective sensitivity increases with a trend similar to the one of the otolith model.

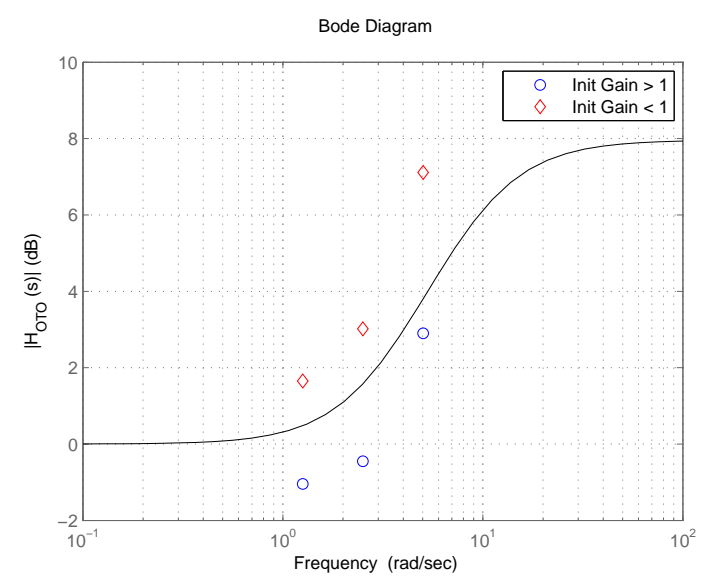

(a) Visual Amplitude $=1 \mathrm{~m} / \mathrm{s}^{2}$

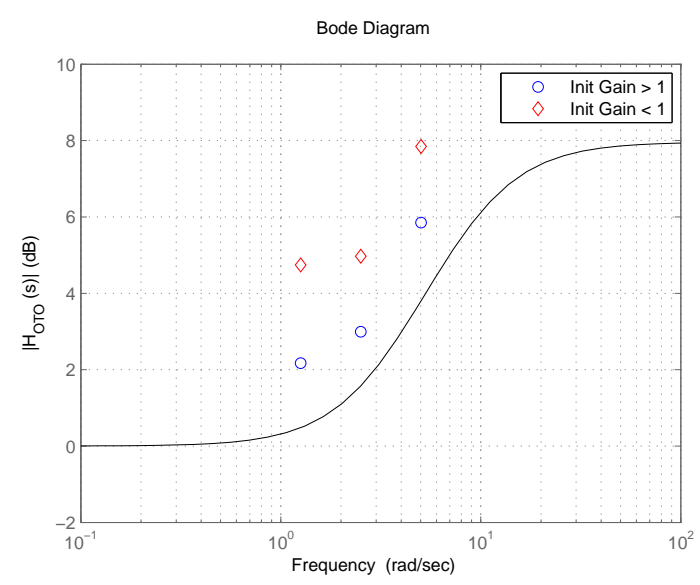

(b) Visual Amplitude $=2.5 \mathrm{~m} / \mathrm{s}^{2}$

Figure 5. Otolith dynamics with sensitivity indication for the conditions with visual amplitude of $1 \mathrm{~m} / \mathrm{s}^{2}$ (left) and conditions with $2.5 \mathrm{~m} / \mathrm{s}^{2}$ (right).

This frequency dependency can be used to improve the design of current MCAs. A motion filter that included an inverse model of the otolith could be used to filter the accelerations that are used as input for the motion platform. This means that high frequency accelerations would be scaled down more than low frequency accelerations. A filter like this decreases the jerk during motion simulations, which might decrease the overestimation of inertial motion normally found in a simulator environment. ${ }^{4}$

However, one should consider in the results the fact that there were reports of a motion artifact being felt especially during the high frequency condition. These motion artifacts are created due to the mechanical limitations of motion platforms when dealing with higher derivatives of the motion signal such as jerk. For 
example, Grant et al. ${ }^{10}$ had to develop a compensation algorithm to eliminate non-linearities created by the motion platform. In our case, 'stick-slip' was detected for the higher frequency motion profiles. Such mechanical artifact will be solved in the near future and it will then be investigated whether this could have an influence in the preferred motion gains.

\section{IV.C. Motion gain dependence on initial condition}

Although subjects were asked to find the best motion gain for the displayed visual information, this value was still dependent on the initial inertial condition. The differences found in the initial conditions showed that an initial motion gain higher than one would yield motion gain values higher than the ones obtained from an initial motion gain lower than one. We were expecting to get similar results for the conditions with the same visual amplitude but the results were statistically different. Nevertheless it seems that subjects tended to a sort of inner-coherence zone where the motion already felt optimal. This inner-coherence zone is different than what is referred to as coherence zone ${ }^{14,15}$ in the literature. In coherence zone studies, subjects are asked to adjust the motion gain such that the motion gain is coherent with the visual. In this way, an upper threshold and a lower threshold define the coherence zone. The upper threshold is defined as the highest motion gain one can have before the motion cue is perceived incongruent when compared with the visual cue. In contrast, the lower threshold is defined as the minimal motion gain one can have before the visual and motion cues are perceived as incongruent. In this study, we did not ask subjects to look for this boundary that divides congruent cues from incongruent cues but for the optimal value between the motion information and visual information. Therefore, we define the inner-coherence zone by an upper and lower threshold that are perceptually indistinguishable from each other when these are measured from different initial inertial amplitudes. We expect the optimal gain to be within the inner-coherence zone. Figure 6 illustrates the concept of inner-coherence zone.

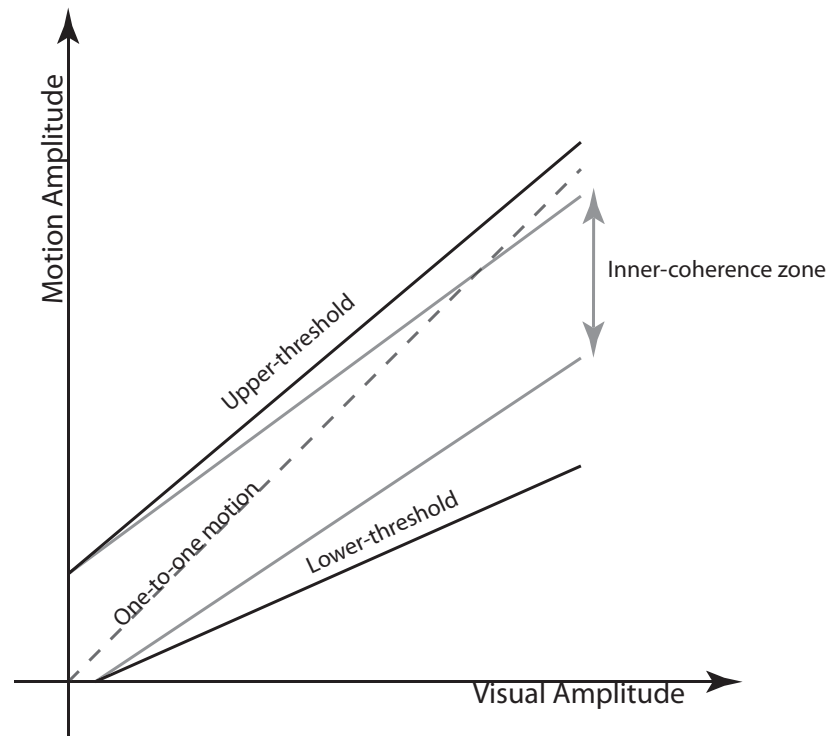

Figure 6. Schematic of the Inner-coherence zone for a simulation environment. The upper boundary of the innercoherence zone crosses the one-to-one motion line suggesting that for higher amplitudes, the optimal motion gain zone needs a motion gain lower than one. This trend was found during this experiment.

\section{Conclusion}

With this experiment we showed that in the Desdemona simulation environment, the optimal motion gain is not one. This was already observed in other studies where a one-to-one inertial/visual ratio was not the preferred motion condition. We conclude that the subjective motion gain depends of the amplitude and the frequency of the stimuli. We found that the preferred motion gain decreases with the increase of the stimuli amplitude. However, we need more data points to define the exact nature of this relation. The results also showed that the motion gain decreases with the increase of the motion frequency. This increase seems to follow the same trend of the otolith models that include jerk sensitivity, like the model of Hosman. ${ }^{9}$ 
Contrary to initial expectations, we found that the optimal gain depended on the initial motion gain. When the initial motion cue was higher than the visual cue, the subjective motion gain was higher than in the conditions where the initial motion cue was smaller than the visual cue.

This study showed that there are still improvements that can be made to the MCAs of current motion simulators. To date, the motion gain in a classical MCA is constant. However, the results showed that a dynamic motion gain algorithm would improve the perception of motion cues in the simulator. By using such an algorithm, we expect to improve the subjective realism of motion simulation and decrease the used motion envelope.

\section{Acknowledgments}

The authors wish to thank Ana R. Valente Pais for helping during the definition of the experimental protocol and the design of the online tuning algorithm.

\section{References}

\footnotetext{
${ }^{1}$ Feenstra, P., Wentink, M., Correia Grácio, B. J., and Bles, W., "Effect of Simulator Motion Space on Realism in the Desdemona Simulator," DSC 2009 Europe, Monaco, 2009.

${ }^{2}$ Pretto, P., Nusseck, H. G., Teufel, H. J., and Bülthoff, H. H., "Effect of lateral motion on driver's performance in the MPI motion simulator," DSC 2009 Europe, Monaco, 2009.

${ }^{3}$ Groen, E. L., Clari, M. S. V. V., and Hosman, R. J. A. W., "Evaluation of perceived motion during a simulated takeoff run," Journal of Aircraft, Vol. 38, No. 4, 2001, pp. $600-606$.

${ }^{4}$ Groen, E. L., Clari, M. S. V. V., and Hosman, R. J. A. W., "Perception model analysis of flight simulator motion for a decrab maneuver," Journal of Aircraft, Vol. 44, No. 2, 2007, pp. 427 - 435.

${ }^{5}$ Wentink, M., Valente Pais, A. R., Mayrhofer, M., Feenstra, P., and Bles, W., "First Curve Driving Experiments in the Desdemona Simulator," DSC 2008 Europe, Monaco, January-February 2008.

${ }^{6}$ Valente Pais, A. R., Wentink, M., van Paassen, M. M., and Mulder, M., "Comparison of Three Motion Cueing Algorithms for Curve Driving in an Urban Environment," PRESENCE: Teleoperators and Virtual Environments, Vol. 18, 2009, pp. 200 221 .

${ }^{7}$ Guedry, F. E., Psychophysics of Vestibular Sensation, Handbook of Sensory Physiology VI, The Vestibular System part 2, New York: Springer-Verlag, 1974.

${ }^{8}$ Roza, M., Wentink, M., and Feenstra, P., "Performance Testing of the Desdemona Motion System," AIAA Modeling and Simulation Technologies Conference and Exhibit, 2007.

${ }^{9}$ Hosman, R. J. A. W., Pilot's perception and control of aircraft motions, Ph.D. thesis, Faculty of Aerospace Engineering, Delft University of Technology, 1996.

${ }^{10}$ Grant, P. R. and Haycock, B., "Effect of Jerk and Acceleration on the Perception of Motion Strength," Journal of Aircraft, Vol. 45, No. 4, 2008, pp. $1190-1197$.

${ }^{11}$ Naseri, A., Grant, P. R., and Dufort, P., "Modeling the Perception of Acceleration and Jerk using Signal Detection Theory," AIAA Modeling and Simulation Technologies Conference and Exhibit, 2008.

${ }^{12}$ Soyka, F., Teufel, H. J., Beykirch, K. A., Robuffo Giordano, P., Butler, J. S., Nieuwenhiuzen, F. M., and Bülthoff, H. H., "Does jerk have to be considered in linear motion simulation?" AIAA Modeling and Simulation Technologies Conference and Exhibit, 2009.

${ }^{13}$ Wentink, M., Bos, J., Groen, E. L., and Hosman, R. J. A. W., "Development of the Motion Perception Toolbox," AIAA Modeling and Simulation Technologies Conference and Exhibit, 2006.

${ }^{14}$ van der Steen, H., Self-Motion Perception, Ph.D. thesis, Delft University of Technology, 1998.

${ }^{15}$ Valente Pais, A. R., van Paassen, M. M., Mulder, M., and Wentink, M., "Perception Coherence Zones in Flight Simulation," AIAA Modeling and Simulation Technologies Conference and Exhibit, 2009.
} 